Revue

de Ihistoire des religions
Revue de l'histoire des religions

2| 2021

La sacralisation de figures païennes à la fin de l'Antiquité (IIe-V|e s.) : poètes, philosophes, hiérophantes et prophètes

\title{
La sacralisation de figures païennes à la fin de
}

l'Antiquité

Avant-propos

Lucia Maddalena Tissi et Philippe Hoffmann

\section{OpenEdition}

Édition électronique

URL : https://journals.openedition.org/rhr/11189

DOI : 10.4000/rhr.11189

ISSN : 2105-2573

Éditeur

Armand Colin

Édition imprimée

Date de publication : 15 juin 2021

Pagination : 187-205

ISBN : 978-2-200-93376-0

ISSN : 0035-1423

Référence électronique

Lucia Maddalena Tissi et Philippe Hoffmann, « La sacralisation de figures païennes à la fin de I'Antiquité », Revue de l'histoire des religions [En ligne], 2 | 2021, mis en ligne le 15 juin 2021, consulté le 02 juillet 2021. URL : http://journals.openedition.org/rhr/11189; DOI : https://doi.org/10.4000/rhr 11189 


\section{LUCIA MADDALENA TISSI \\ PHILIPPE HOFFMANN}

Laboratoire d'excellence

"Histoire et anthropologie des savoirs, des techniques et des croyances»

Laboratoire d'études sur les monothéismes

École Pratique des Hautes Études

Paris-Sciences-Lettres

\section{La sacralisation de figures païennes à la fin de l'Antiquité Avant-propos}

Ce numéro thématique porte sur une question centrale dans la construction de l'identité culturelle à la fin de l'Antiquité, celle de la sacralisation. La figure de l'homme divin de l'époque, le $\theta \varepsilon i ̃ o \varsigma$ $\grave{\alpha} v \eta ́ \rho$, a été étudiée et analysée par les savants et mise en relation avec son homologue chrétien, le saint - comme l'ont souligné le célèbre essai de Peter Brown, publié en 1971, et d'autres textes dans son sillage ${ }^{1}$. Nous nous proposons ici non pas de retracer

1. Sur la vie des moines et des ascètes de l'Antiquité tardive et sur la fonction sociale et politique de l'homme divin, voir : Peter Brown, « The Rise and Function of the Holy Man in Late Antiquity », The Journal of Roman Studies, t. 61, 1971, p. 80-101, et Id., "The Rise and Function of the Holy Man in Late Antiquity, 1971-1997 », Journal of Early Christian Studies, t. 6, 1998, p. 335-376. Dans ce dernier essai, P. Brown introduit une distinction entre l'homme divin chrétien, qui

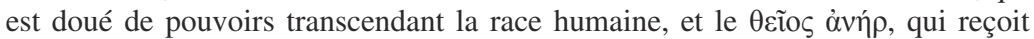
son pouvoir de et dans la société. Sur le rôle du philosophe païen dans l'Antiquité tardive et sur son autorité sociale, voir P. Brown, « The Philosopher and Society in Late Antiquity », The Center for Hermeneutical Studies in Hellenistic and Modern Culture, t. 34, 1980, p. 1-17. Concernant l'homme divin en général dans l'Antiquité tardive, voir : Ludwig Bieler, $\Theta E I O \Sigma$ ANHP. Das Bild des "göttlichen Menschen" in Spätantike und Frühchristentum, t. 1-2, Vienne, Buchhandlung Oskar Höfels, 1935-1936 ; Garth Fowden, "The Pagan Holy Man in Late Antique Society », The Journal of Hellenic Studies, t. 102, 1982, p. 33-59 ; Graham Anderson, Sage, Saint and Sophist. Holy Men and Their Associates in the Early Roman Empire, Londres/New York, Routledge, 1994. Sur la fonction éducatrice de l'homme divin, 
l'histoire de cette figure, ce qui a déjà été fait par le passé, mais d'esquisser, à travers des études de cas, une galerie de portraits de figures païennes de l'Antiquité qui ont été sacralisées et parfois insérées dans une chaîne de savoir(s)2 .

La période à laquelle nous nous intéressons, qui va du $\mathrm{II}^{\mathrm{e}}$ au $\mathrm{VI}^{\mathrm{e}}$ siècle ${ }^{3}$, est, en effet, marquée à la fois par une continuité et une fracture entre deux «structures » théologiques - «païenne» et chrétienne -, qui se réalisent de diverses manières. Par ailleurs, l'allusion à cette convergence et/ou divergence, ou mieux, à l'interrelation culturelle et dynamique entre différents systèmes, est un élément clef pour explorer les contextes dans lesquels cette sacralisation intervient et la méthode employée par nos sources ${ }^{4}$.

voir : Robert Kirschner, «The Vocation of Holiness in Late Antiquity », Vigiliae Christianae, t. 38, 1984, p. 105-124 ; Divine Men and Women in the History and Society of Late Hellenism, dir. Maria Dzielska, Kamilla Twardowska, Cracovie, Jagiellonian University Press, 2013. On trouve une bibliographie de synthèse sur ce sujet dans Daniel Lemeni, « Narrating the Holy Man in Late Antiquity : The Case of Shenoute of Atripe », Theologia, t. 66, 2016, p. 142-154 et dans The Oxford Dictionary of Late Antiquity, éd. Oliver Nicholson, Oxford, 2018, p. 735-736.

2. Il n'est pas étonnant de tomber, par exemple, sur l'image d'un philosophe qui porte un nimbe : voir Paul Zanker, Die Maske des Sokrates. Das Bild des Intellektuellen in der antiken Kunst, Munich, Verlag C. H. Beck, 1995, p. 297-298 et p. 298, fig. 172 (l'image date de 395 apr. J.-C.). Le visage de Proclus apparaît entouré d'un nimbe dans Marinus, Proclus ou Sur le Bonheur, éd. Henri Dominique Saffrey et Alain-Philippe Segonds (avec la collaboration de Concetta Luna), Paris, Les Belles Lettres, 2001, § 23, 26-29 ; voir le commentaire des éditeurs p. 144, n. 3.

3. Pour la période appelée, avec beaucoup de prudence, Antiquité tardive, voir Polymnia Athanassiadi, «Antiquité tardive : construction et déconstruction d'un modèle historiographique ", Antiquité tardive, t. 14, 2006, p. 311-324 et Arnaldo Marcone, «A Long Late Antiquity ? Considerations on a Controversial Periodization », Journal for Late Antiquity, t. 1, 2008, p. 4-19.

4. Sur le concept d' 'interaction» culturelle, voir: Frank R. Trombley, Hellenic Religion and Christianization c. 370-529, Leyde, Brill, 1993 ; Gilles Dorival, "Hellénisme et christianisme. Continuités et ruptures », Les chrétiens et l'hellénisme. Identités religieuses et culture grecque dans l'Antiquité tardive, éd. Arnaud Perrot, Paris, Éditions Rue d'Ulm, 2012, p. 21-30 ; Id., "Grecs, Romains, Juifs, Chrétiens en interaction », Recherches de Science Religieuse, t. 101.4, 2013-2014, p. 499-516. Ce terme a désormais été remplacé par celui d' "interrelation", qui relève d'un dialogue entre deux systèmes de pensée différents : voir Judith M. Lieu, Christian Identity in the Jewish and Graeco-Roman World, Oxford/New York, Oxford University Press, 2004, p. 14, et L'oiseau et le poisson. Cohabitations religieuses dans les mondes grec et romain, dir. Nicole Belayche, Jean-Daniel Dubois, Paris, PUPS, 2011, p. 8. 


\section{QU'EST-CE QUE LA SACRALISATION?}

Mais qu'est-ce que la sacralisation des figures païennes antiques $^{5}$ ? Par ce terme, nous désignons ici le phénomène qui conduit à placer certaines figures, tirées soit de l'histoire soit du répertoire mythologique de la tradition gréco-romaine, dans un contexte "sacralisé », c'est-à-dire dans une sphère quasi divine empreinte d'une sorte de ritualité mystérique ${ }^{6}$. Autrement dit, les sages de l'Antiquité auraient transmis une parole révélée, un savoir, ou mieux, des savoirs sacrés.

Qu'il s'agisse de poètes comme Homère, Hésiode et Virgile, de philosophes tels que Pythagore, Platon et Aristote, de figures oscillant entre histoire et fiction, comme celles des Sept Sages? ou encore d'autres figures mythiques et historiques de la tradition grecque $^{8}$ et romaine, comme les Sibylles, ou issues d'autres traditions, comme Zoroastre, on observe à l'époque tardive une sacralisation des figures païennes appartenant aux temps anciens, valorisées selon une antique tradition. On pourrait également parler d'une sorte de divinisation et de «manticisation» de ces figures, qui se voient attribuer un statut divinatoire ou prophétique et qui, dans le contexte chrétien, sont presque sanctifiées. Ce phénomène se réalise sur les plans littéraire et iconographique, la portée de cette sacralisation étant, en effet, visible à plusieurs niveaux.

Ajoutons que certains livres de l'Antiquité sont, pour ainsi dire, à tel point sacralisés, qu'ils deviennent porteurs d'un message

5. Pour une réflexion autour du concept de sacralisation voir ici l'article de Sébastien Morlet.

6. Sur le contexte général, voir de récents travaux : Nicole Belayche, Francesco Massa, Philippe Hoffmann (éd.), Les mystères au II siècle de notre ère : un tournant, Turnhout, Brepols, 2021, et Francesco Massa, Nicole Belayche (dir.), Les philosophes et les mystères dans l'empire romain, Liège, Presses universitaires de Liège, 2021.

7. Cf. Platon, Protagoras, 343a.

8. Ulysse, par exemple, devient le paradigme de l'âme exilée hors de la matière, secouée par les vagues de la vie. Selon Numénius (puis Porphyre), il est l'image de celui qui traverse les différents stades de la génération pour retourner auprès de ceux qui n'ont pas l'expérience de la mer, l'image de l'âme qui revient vers sa véritable patrie (Porphyre, L'antre des nymphes 34). Par ailleurs, Circé, Calypso (e.g. Plotin, Enn. I 6 [1] 8, 18-20 Henry - Schwyzer), les Sirènes (e.g. Clément d'Alexandrie, Protreptique, XII 118, 1-3) symbolisent la vie matérielle et les tentations. Sur Ulysse en tant que sage platonicien chez Maxime de Tyr, voir Félix Buffière, Les mythes d'Homère et la pensée grecque, Paris, Les Belles Lettres, 1956, p. 386-388. 
prophétique et divinatoire et qu'ils sont considérés comme indispensables au développement de la paideia et au progrès moral et spirituel. Il suffit de penser aux Oracles chaldaïques, la «Bible» des néoplatoniciens ${ }^{9}$, les seuls textes avec le Timée qui, aux yeux de Proclus, seraient dignes de rester en circulation ${ }^{10}$. Cette forme de sacralisation se manifeste également dans l'interprétation des Livres Saints comme oracles ${ }^{11}$ et, dans le contexte chrétien, à travers l'interprétation de l'Ancien Testament comme annonce de l'arrivée du Christ, les chrétiens faisant cependant bien la différence entre le véritable oracle chrétien et les faux oracles païens. Pour ne citer qu'un exemple, la source prophétique Castalie, près d'Antioche, est présidée, au cours $\mathrm{du} \mathrm{v}^{\mathrm{e}}$ siècle, selon Eudocie, par un diacre chrétien du nom de Praulio, qui récite le seul véritable oracle, la Bible ${ }^{12}$. Ainsi, nous serions tentés d'affirmer que la voix des livres a la même valeur que celle des figures sacralisées. Par ailleurs, les paroles de figures d'autorité comme Platon, Aristote et Pythagore, et celles des anciens théologiens (e.g. Orphée, Homère) ${ }^{13}$ sont exploitées par le néoplatonisme tardif ; le statut quasi sacré de leurs auteurs

9. À propos de l'association des Oracles chaldaïques à la Bible, proposée par Franz Cumont et par d'autres savants, voir Helmut Seng, Les Oracles chaldaïques. Un livre sacré de l'Antiquité tardive, Turnhout, Brepols, 2016, p. 19.

10. Marinus, Proclus, 38, 16-20 Saffrey - Segonds - Luna. Certains textes de Platon sont également revêtus d'un statut prophétique, comme le déclare Olympiodore à propos du Timée, de la République, du Phèdre et du Théétète, qui présentent aux hommes quatre typologies d'enthousiasme (Olympiodore, In Alc. 1, 3-2, 13 Westerink).

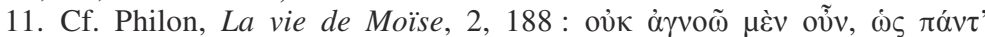

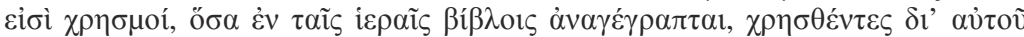
( Certes, je n'ignore pas que tout ce qui se trouve dans les Livres Saints est oracles rendus par lui », Philon d'Alexandrie. De Vita Mosis I-II, introduction, traduction et notes par Roger Arnaldez, Paris, éd. du Cerf, 1967). Sur les trois catégories de prophéties chez Philon, voir Giulia Sfameni Gasparro, Oracoli profeti sibille: rivelazione e salvezza nel mondo antico, Rome, Libreria Ateneo Salesiano, 2002, p. 63-64.

12. Eudocie, La Vie de saint Cyprien, 12-19 Bevegni. Voir Gianfranco Agosti, «La conversione della fonte Castalia in un pannello del mosaico della chiesa di Qasr el-Lebia ", Des Géants à Dionysos. Mélanges offerts à Francis Vian, dir. Domenico Accorinti et Pierre Chuvin, Alexandrie, Edizioni dell'Orso, 2003, p. 541-564.

13. Luc Brisson, Orphée et l'orphisme dans l'Antiquité gréco-romaine, Aldershot, Variorum, 1995; Robert Lamberton, Homer the Theologian. Neoplatonist Allegorical Reading and the Growth of the Epic Tradition, Berkeley, University of California Press, 1986. 
respectifs n'empêche cependant pas des révisions exégétiques de leur pensée ${ }^{14}$.

\section{Deux Philosophes Sacr(alis)és : Pythagore et Platon}

Cet intérêt pour les sages du passé, qui jouissent d'un prestige et d'une autorité sacrée dans le vaste champ de la paideia ${ }^{15}$ et de la philosophie, s'inscrit dans un effort d'harmonisation ( $\sigma \nu \mu \varphi \omega v i ́ \alpha)$ chez les représentants de la pensée philosophique tardive. Et par qui aurait-on pu commencer à explorer les portraits des sages, si ce n'est par les philosophes et poètes par excellence de la pensée hellène, à savoir Pythagore et Platon d'un côté, Homère et Hésiode de l'autre?

Commençons par les deux premiers. Pythagore et Platon sont considérés comme les prototypes des hommes divins, comme des modèles philosophiques intégrés à une chaîne de savants et grâce auxquels la philosophie s'est transmise au fil des siècles. Leur sacralisation, qui porte sur leur fonction paradigmatique, permet de les élever à un rang presque divin. Le philosophe néoplatonicien Isidore d'Alexandrie (fin du $v^{\mathrm{e}}$ siècle), par exemple, compare les deux philosophes à des âmes ailées qui habitent les régions supracélestes $^{16}$.

14. Voir ici l'article d'Adrien Lecerf.

15. Les philosophes anciens sont des guides pour la paideia puisqu'ils ont eux-mêmes pris les dieux pour guides, selon l'empereur Julien (Julien, Lettre 89b [300d], p. 168, 18-21 Bidez).

16. Damascius, Hist. phil. 34D Athanassiadi (Ep. Phot. 36 et fr. 77 Zinzten) :

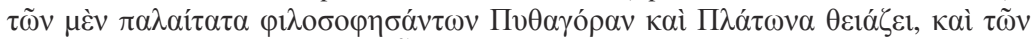

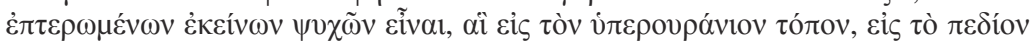

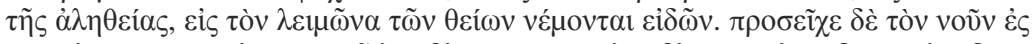

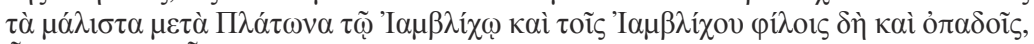

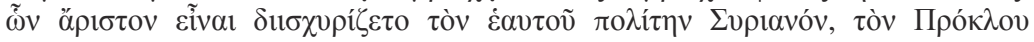

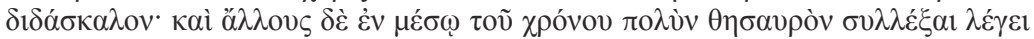

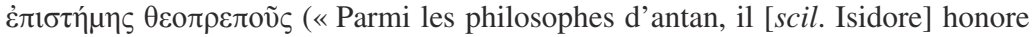
Pythagore et Platon comme êtres divins, et il [considère] qu'ils étaient de ces âmes ailées qui atteignent au lieu supra-céleste, dans la plaine de la Vérité, dans la prairie des formes divines. Après Platon, il s'attachait surtout à Jamblique et aux amis et adeptes de Jamblique, parmi lesquels le meilleur - affirmait-il avec vigueur était son compatriote Syrianus, le maître de Proclus. Il dit qu'entretemps il y en avait d'autres qui avaient accumulé un riche trésor de connaissance véritablement divine »). Le lieu supra-céleste et la plaine de la Vérité font allusion au Phèdre de Platon (respectivement $247 \mathrm{c} 3$ et $248 \mathrm{~b} 6$ ). 
Pythagore, l'initiateur de la pensée grecque, est considéré comme un homme divin, mais aussi comme un oracle. La figure de Pythagore, suspendue entre histoire et légende, a en effet représenté un modèle non seulement de sagesse et de conduite, mais aussi d'actions miraculeuses et thaumaturgiques associées à des aptitudes prophétiques ${ }^{17}$. Son succès à l'époque impériale, amorcé par la Vie d'Apollonios de Tyane écrite par Philostrate, se manifeste dans les portraits biographiques de Diogène Laërce ${ }^{18}$, de Porphyre (Vie de Pythagore) et de Jamblique (Vie de Pythagore ou Sur le mode de vie pythagoricien). En vertu de son lien avec le dieu oraculaire Apollon ${ }^{19}$, Pythagore dispense son enseignement par le biais d'une communication symbolique, à la manière des oracles apolliniens ${ }^{20}$. Il s'agit d'énigmes - $\gamma \rho \tilde{\imath} \varphi \mathrm{or}$ - en apparence obscures $^{21}$, mais transparentes et claires pour ceux qui possèdent

17. Sur Pythagore voir Constantin Macris, «Pythagore de Samos», Dictionnaire des philosophes antiques, dir. Richard Goulet, t. VII, Paris, CNRS Éditions, 2018, p. 681-850.

18. Voir Diogène Laërce, Vies et doctrines des philosophes illustres, VIII 1-50 Dorandi.

19. Pythagore possède, en effet, le même statut que le dieu oraculaire Apollon (il est l'incarnation d'Euphorbe) et il est dès lors associé à cette divinité, comme le prouvent plusieurs détails : le nom de sa mère qui, à la suite d'un oracle de la Pythie à Delphes, fut changé de Parthénis en Pythaïs (Jamblique, Vie de Pythagore, II 4, p. 7, 19 Deubner) ; l'épithète de « fils d'Apollon », d'après un poète de Samos (ibid., II 5, p. 7, 6-7 Deubner et Porphyre, Vie de Pythagore, 2, p. 37, 12-13 des Places) ; l'étymologie du nom de Pythagore, « celui qui a été annoncé par la Pythie (ou le dieu Pythien)» (Jamblique, Vie de Pythagore, II 7, p. 7, 22) ou encore comme celui qui annonce la vérité à l'égal du dieu Pythien (Diogène Laërce, Vies et doctrines des philosophes illustres, VIII 21, p. 612, 234-237 Dorandi : c'est l'opinion d'Aristippe de Cyrène) ; l'association entre Pythagore et Apollon Hyperboréen (Jamblique, Vie de Pythagore, XXVIII 140, p. 79, 13-16 Deubner ; Ael. VH II 26 ; Diogène Laërce, Vies et doctrines des philosophes illustres, VIII 11, p. 606, 116-118 Dorandi). Voir aussi le lien entre Abaris et Pythagore (Jamblique, Vie de Pythagore, XIX 91, p. 53, 7 - 94 p. 55, 5 Deubner). Selon Jamblique, l'âme de Pythagore est divine et constitue une sorte de médiation entre le divin et l'humain (Jamblique, Vie de Pythagore, II 8, p. 7, 28 - p. 8, 8 Deubner).

20. Jamblique, Vie de Pythagore, XXIX 161, p. 90, 25 - 91, 5 Deubner.

21. Les symboles sont révélés grâce à une exégèse qui clarifie leur message, à la manière des prophéties et des oracles d'Apollon Pythios (Jamblique, Vie de Pythagore, XXIII 105, p. 60, 22 - p. 61, 4 Deubner). Pythagore ne prononce cependant pas d'oracles adressés aux malfaiteurs (Jamblique, Vie de Pythagore, XXVII 133, p. $75,13-24$, XXX 177, p. 99, 12-14 et XXXI 214, p. 116, 19-21 Deubner). Il faut remarquer que la divination est très importante chez les pythagoriciens (Jamblique, Vie de Pythagore, XXIX 163, p. 92, 5 Deubner). En outre, les préceptes adoptés par les pythagoriciens sont comme des oracles de la Pythie (Jamblique, Vie de Pythagore, XXXI 213, p. 116, 1-5 Deubner ; voir aussi 
la connaissance et donc la capacité de les résoudre ${ }^{22}$. Or, cette association entre sentences et oracles se retrouve également dans les recueils des sentences $(\dot{\alpha} \pi \circ \varphi \theta \dot{\varepsilon} \gamma \mu \alpha \tau \alpha)$ des Sept Sages : les oracles sont désormais des maximes éthiques. La sagesse de Pythagore constitue donc un autre maillon, le premier, de la chaîne de savoirs/savants ${ }^{23}$; le philosophe semble d'ailleurs avoir tiré une partie de sa connaissance des Sept $\mathrm{Sages}^{24}$, d'Orphée ${ }^{25}$, d'Aglaophamos ${ }^{26}$, des Magi, des mystères d'Éleusis ${ }^{27}$ ainsi que des Égyptiens, des Chaldéens, des Phéniciens, des Hébreux et des Arabes $^{28}$.

les oracles d'Apollon sur Phalaris, Vie de Pythagore, XXXII 221, p. 119, 19-222, p. 120, 11 Deubner) ; une maxime (àró $\varphi \theta \varepsilon \gamma \mu \alpha)$ de Pythagore est comparée à un oracle divin (Jamblique, Vie de Pythagore, VII 34, p. 20, 9-12 Deubner). Sur la question générale des «symboles » pythagoriciens (et des maximes des Sept Sages), voir récemment $\mathrm{Ph}$. Hoffmann, "Maximes, proverbes et formes brèves dans la philosophie grecque», dans les Actes du XXX colloque de la Villa Kérylos : Les petites phrases. Puissance de la brièveté dans les littératures d'Orient et d'Occident (Institut de France-AIBL, 11-12 octobre 2019), Cahiers de la Villa «Kérylos », n 31, Paris 2020, p. 13-89, spéc. p. 29-66 (avec bibliographie).

22. Jamblique, Vie de Pythagore, XXXIV 247, p. 132, 23 - p. 133, 2 Deubner. Pythagore contourne l'autorité d'Apollon (Jamblique, Vie de Pythagore, XXXV 261, p. 140, 19 Deubner), à tel point que, à quelqu'un qui lui demandait de lui expliquer la cause de certains faits, Pythagore répondit qu'Apollon, lorsqu'il vaticine, ne donne jamais de raisons (Jamblique, Vie de Pythagore, XXX 177 , p. $99,14-18$ Deubner).

23. D'autres philosophes sont également sacralisés, par exemple Jamblique, que Julien considère comme le troisième philosophe divin après Pythagore et Platon. Dans une lettre, Julien demande au philosophe Priscus le commentaire des Oracles chaldaïques réalisé par Jamblique (Iul. Ep. 12 [425d], p. 12, 7-14 Bidez).

24. Jamblique, Vie de Pythagore, XVIII 83, p. 48, 6-13 Deubner.

25. La figure d'Orphée, tout comme celles des Chaldéens, joue en réalité un rôle de pivot dans la transmission de la sagesse. Orphée est décrit comme le plus ancien des philosophes qui ont inspiré l'empereur Julien (Julien, Or. VII [215B], p. 58,

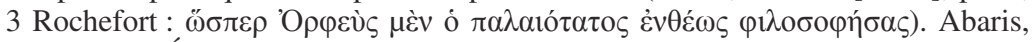
Empédocle, Épiménide, par exemple, sont d'autres figures de sages divins : cf. Jamblique, Vie de Pythagore, XXVIII 135 Deubner ; Porphyre, Vie de Pythagore, 29 p. 49, 19-26 des Places. Sur Pythagore, voir ici l'article de Fabienne Jourdan.

26. Christian August Lobeck, Aglaophamus, sive de theologiae mysticae Graecorum causis, Königsberg (Kaliningrad), 1829; L. Brisson, "Orphée, Pythagore et Platon. Le mythe qui établit cette lignée », Metaphysik und Religion. Zur Signatur des spätantiken Denkens. Akten des Internationalen Kongresses vom 13.-17. März 2001 in Würzburg, dir. Theo Kobusch et Michael Erler, MunichLeipzig, K.G. Saur, 2002, p. 416-427.

27. Jamblique, Vie de Pythagore, XXVIII 151, p. 85, 14-20 Deubner.

28. Porphyre, Vie de Pythagore, 11, p. 41.7-9 des Places. Il s'agit des peuples sages. D'après Celse, il faut compter parmi les peuples ěv $\theta \varepsilon o 1$, inspirés par la divinité, les Chaldéens, les Mages, les Égyptiens, mais pas les Hébreux (Orig. 
L'image de Platon comme figure sacrée, assimilée à la divinité, est largement reprise dans la pensée des philosophes néoplatoniciens. Pour ces philosophes, il existe, en effet, une véritable chaîne de savoirs, ou mieux, de savants, dont l'objectif est de créer une symphonie de la pensée, comme le résume parfaitement le titre du traité (aujourd'hui perdu) de Syrianus sur «L'accord d'Orphée,

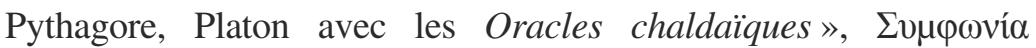

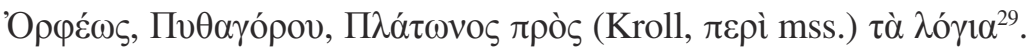
Dans son introduction à la Théologie platonicienne Proclus évoque un chœur divin qui transmet la mystagogie platonicienne en employant un langage marqué par l'influence des mystères d'Éleusis et des oracles divins. «Toute la théologie grecque »- déclare-t-il « est fille de la mystagogie d'Orphée. Pythagore le premier avait appris d'Aglaophamos ${ }^{30}$ les initiations relatives aux dieux, Platon, ensuite, a reçu des écrits pythagoriciens et orphiques la science toute parfaite qui les concerne $»^{31}$ : la philosophie est donc, pour ainsi dire, une théologie révélée ${ }^{32}$. Les sages qui se transmettent ce savoir appartiennent, dès lors, à une race sacrée, à une véritable chaîne

Cels. VI 80, cf. VIII, 69). Le grief implicite est qu'ils rejettent les coutumes ancestrales des autres peuples, au profit de leur seul «Très-Haut ». Concernant la sagesse barbare des prêtres égyptiens vue par les Grecs voir Sydney H. Aufrère, "Sous le vêtement de lin du prêtre isiaque, le "philosophe". Le "mythe" égyptien comme Sagesse barbare chez Plutarque », Alexandrie la divine. Sagesses barbares. Échanges et réappropriations dans l'espace culturel gréco-romain, dir. Charles Méla, Frédéric Möri et alii, Genève, La Baconnière, 2016, p. 191-270.

29. Suda $\sigma 1662, \pi 2473$ Adler. Voir H. D. Saffrey, A.-Ph. Segonds, Marinus. Proclus, p. 140 n. 8 et H.D. Saffrey, «Accorder entre elles les traditions théologiques : une caractéristique du néoplatonisme athénien », On Proclus and his Influence in Medieval Philosophy, dir. Egbert Peter Bos, Pieter Ane Meijer, Leyde, Brill, 1992, p. 35-50 = Id., Le néoplatonisme après Plotin, Paris, Vrin, 2000, p. 143-158.

30. Voir L. Brisson, « Orphée, Pythagore et Platon. Le mythe qui établit cette lignée ».

31. Proclus, Théologie Platonicienne, I 5, p. 25, 26-p. 26,4 Saffrey-Westerink :

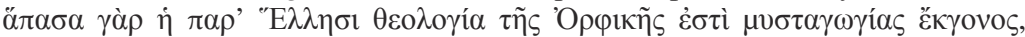

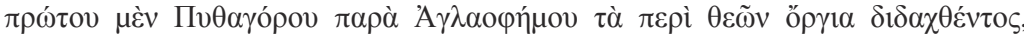

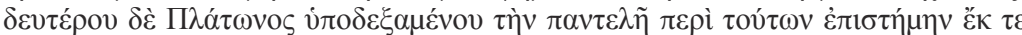

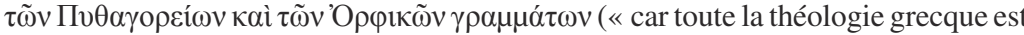
fille de la mystagogie d'Orphée : Pythagore le premier avait appris d'Aglaophamos les initiations relatives aux dieux, Platon ensuite a reçu des écrits pythagoriciens et orphiques la science toute parfaite qui les concerne», trad. des éditeurs).

32. Sur ce sujet, voir le volume Exégèse, révélation et formation des dogmes dans l'Antiquité tardive, dir. Alain Le Boulluec, Luciana Gabriela Soares Santoprete, Andrei Timotin, Paris, Institut d'études augustiniennes, 2020. 
d'or ${ }^{33}$, pour reprendre la célèbre image homérique ${ }^{34}$, leur statut étant comparable à celui des mystagogues et des prophètes, autrement dit aux personnes inspirées par Dieu et qui parlent en son nom. Cette sacralisation intervient, par ailleurs, dans un contexte culturel où le philosophe est représenté comme un «homme divin", souvent en association avec le hiérophante qui dévoile des objets sacrés dans le cadre des rituels mystériques ${ }^{35}$. Par conséquent, le maître, qui guide ses élèves tout au long de leur parcours initiatique et mystérique de connaissance, propose une exégèse des textes, un acte inspiré comparable à celui d'un prophète. Le philosophe représente ainsi le «hiérophante du monde entier $»^{36}$. Dans le cadre de cette vision théosophique, la fonction des oracles divins devient essentielle. En effet, comme l'explique l'Empereur Julien dans son Discours à la Mère des dieux ${ }^{37}$, les théories d'Aristote sont incomplètes à moins d'être harmonisées avec celles de Platon et avec les oracles divins. En outre, le philosophe Hiéroclès ( $v^{\mathrm{e}}$ siècle) aurait harmonisé, dans le quatrième livre de son ouvrage Sur la providence et le destin, les dogmes de Platon avec les $\lambda$ ó $1 \alpha$ et les rites sacrés ${ }^{38}$. Cette

33. Cf., par exemple, Damascius, Hist. Phil. 98E Athanassiadi (Ep. Phot. 151 ; p. 206 Zintzen).

34. Homère, Iliade VIII, 19.

35. E.g. Porphyre, Vie de Plotin 15, 1-6 Henry - Schwyzer ; Marinus, Proclus 19, 25-30 Saffrey - Segonds - Luna.

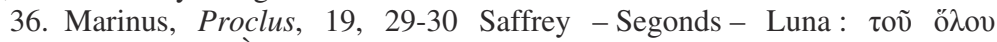

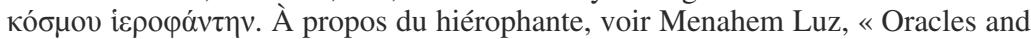
Revelations as a Philosophical Mannerism », Exégèse, révélation et formation des dogmes dans l'Antiquité tardive, p. 37-43; et Dominique Jaillard, " "Le philosophe ne doit pas être le prêtre d'une seule cité... Il doit être universellement le hiérophante du monde entier " (Marinus, Proclus ou Sur le bonheur, 19) », Les philosophes et les mystères dans l'empire romain, cité supra (note 6), p. 171-191, et, dans le même volume (p. 193-203), Ph. Hoffmann, «Le néoplatonisme tardif et les mystères. Quelques jalons ».

37. Julien, Sur la mère des dieux 3, p. 10, 6-11 Ugenti (VIII [V] 3, 162c4-d1 Rochefort). Sur Aristote témoin de petits mystères, par rapport à Platon qui est témoin de grands mystères, et sur la mise en relation avec le cursus studiorum des élèves de philosophie, cf. Marinus, Proclus, 13 Saffrey - Segonds - Luna et le commentaire des éditeurs p. 109 n. 2. Voir également Joseph Bidez, Un singulier naufrage littéraire dans l'antiquité. À la recherche des épaves de l'Aristote perdu, Bruxelles, Office de publicité, 1943, p. 30. Sur les cursus au sein de l'École néoplatonicienne d'Alexandrie, voir Ph. Hoffmann, «Le cursus d'étude dans l'École néoplatonicienne d'Alexandrie », Alexandrie la Divine, vol. I, p. 342-353.

38. Hiéroclès ap. Photius, Bibliothèque, 214, 173a : ó $\delta \varepsilon \bar{\varepsilon} \delta^{\prime} \tau \alpha \grave{\alpha} \lambda \varepsilon \gamma o ́ \mu \varepsilon v \alpha \lambda$ ó$_{1} \alpha$

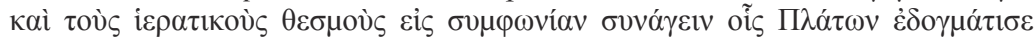
$\beta$ ov́ $\lambda \varepsilon \tau \alpha$. Il est probablement question des Oracles chaldä̈ques et des rites théurgiques : voir Constantin Macris, «Hiéroclès d'Alexandrie », Alexandrie la 
association entre philosophie et oracles témoigne de la fonction médiatrice du philosophe, entre le monde des hommes et le monde divin, et de son statut mantique.

\section{PoÈtes divins et ORACLeS : HoMÈre, Hésiode ET Virgile}

Quant à l'autre couple de sages sacralisés, maîtres de théologie, il s'agit des poètes Homère et Hésiode. Ces derniers représentent, tout au long de l'Antiquité, les racines les plus anciennes de la littérature grecque et la base de la paideia dans le monde hellénophone, et sont en outre considérés comme des prophètes ${ }^{39}$. L'empereur Julien ${ }^{40}$ explique comment les deux poètes seraient animés par une inspiration divine, à la manière des prophètes possédés par la divinité qui les conduit vers la vérité. La valeur quasi magique de leurs vers est également attestée dans la ritualité pythagoricienne: pour les pythagoriciens, réciter les vers d'Homère et d'Hésiode corrige et adoucit les âmes ${ }^{41}$. On peut également évoquer le succès, à l'époque impériale, des Homeromanteia, qui consistent à reprendre les vers homériques en leur attribuant une fonction divinatoire ${ }^{42}$. La parole d'Homère,

Divine, vol. I, p. 384-387, ici p. 386. Sur Hiéroclès, voir entre autres publications de Ilsetraut Hadot, son livre devenu classique, Le Problème du néoplatonisme alexandrin. Hiéroclès et Simplicius, Paris, Études Augustiniennes, 1978.

39. Sur Homère et Hésiode comme maîtres des sciences et de la théologie, voir F. Buffière, Les mythes d'Homère, et R. Lamberton, Homer the Theologian.

40. Julien, Sur le roi Soleil, 11, p. 114, 1-5 Prato (XI [IV] 11, 136b6-c2 Lacombrade) et ibid. 31, p. 146.13-14 Prato (XI [IV] 149c10-12 Lacombrade).

41. Jamblique, Vie de Pythagore, XXIX 164, p. 92, 20-22 Deubner, et Porphyre, Vie de Pythagore, 32, p. 51, 10-11 des Places. Cf. récemment Ph. Hoffmann, «Les prières en prose de Simplicius, entre rhétorique et théologie », Théories et pratiques de la prière à la fin de l'Antiquité, éd. Ph. Hoffmann et Andrei Timotin, Turnhout, 2020, p. 209-267 (p. 236-237).

42. Athanassia Zografou, «Un oracle homérique de l'Antiquité tardive : un livre-miniature à usage oraculaire », Kernos, t. 26, 2013, p. 173-190; Raquel Martín-Hernández, « Using Homer for Divination : Homeromanteia in Context », CHS Research Bulletin, t. 2, 2013, voir en ligne: http://www.chs-fellows. org/2014/03/28/using-homer-for-divination-homeromanteia-in-context/\#_ftn34 ; Crystal Addey, Divination and Theurgy in Neoplatonism. Oracles of the Gods, Farnham, Ashgate, 2014, p. 71-75. Sur les Homeromanteia en tant que « books with pedigrees could be plausibly viewed as direct links to the supernatural worlds » : Mary Beard, "Writing and Religion: Ancient Literacy and the Function of the Written World in Roman Religion », Literacy in the Roman World, dir. John H. Humphrey, Ann Arbor, University of Michigan [« Journal of Roman Archaeology. 
comme plus tard celle de Virgile, diffuse donc une vérité sacralisée, comme en témoignent les Sortes Homericae et les Sortes Vergilianae ${ }^{43}$. Il s'agit d'une sorte de divinisation très répandue parmi les philosophes néoplatoniciens ${ }^{44}$ : à l'instar de la Bible et de la Torah, l'ouvrage d'Homère est considéré comme sacré et comme un mode de communication divine. Les portraits de ces sages sacrés se retrouvent aussi dans l'iconographie. Il n'est donc pas étonnant que soit évoquée, dans la description des thermes du Zeuxippe présentée par Christodore de Coptos $\left(\mathrm{v}^{\mathrm{e}}\right.$ $\mathrm{VI}^{\mathrm{e}}$ siècles), la statue d'Homère, décrite comme un bronze vivant et inspiré, ainsi que celle d'Hésiode, qui semble s'animer pour nous faire entendre un chant inspiré 45 .

\section{LA « CONVERSION « DES SAGES PAÏENS »}

Loin d'être l'apanage des Hellènes, cette sacralisation des figures païennes est également attestée - comme nous l'avons évoqué chez les auteurs chrétiens ${ }^{46}$. Il s'agit alors de se les approprier, de les réinterpréter et de les placer dans un nouveau contexte culturel,

Supplementary series », t. 3], 1991, p. 33-58. Des exemples de PGM IV et VII mentionnent des oracles de Zeus Belos délivrés sous forme de vers d'Homère.

43. Voir Pieter W. van der Horst, « Sortes: Sacred Books as Instant Oracles in Late Antiquity », The Use of Sacred Books in Ancient World, dir. Leonard Victor Rutgers et alii, Louvain, Peeters, 1998, p. 143-174, ici p. 159; Aude Busine, «Usages divinatoires du livre: une 'anti-exégèse'? ", Exégèse, révélation et formation des dogmes dans l'Antiquité tardive, p. 297-310; sur la sacralisation d'Homère, voir aussi F. Buffière Les mythes d'Homère, p. 10-13 et 25-31. Cf. aussi les Sortes Astrampichi et les Sortes Sangallenses. Dans un ostrakon/papyrus couvert d'exercices scolaires, provenant de Karanis (Égypte) et datant du III ${ }^{\mathrm{e}}$ siècle

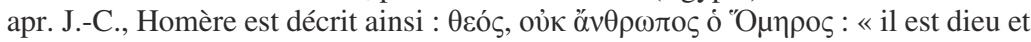
non pas homme, Homère $\gg(O$. Mich. 3.1100, 10-11 = TM 64126 : voir Herbert C. Youtie, John G. Winter, Michigan Papyri, VIII: Papyri and Ostraca from Karanis, second series, Ann Arbor, University of Michigan, 1951, p. 206.

44. R. Lamberton, Homer the Theologian, p. 1-43 et Id., " The Neoplatonists and the Spiritualization of Homer », Homer's Ancient Readers. The Hermeneutics of Greek Epic's Earliest Exegetes, dir. R. Lamberton, John J. Keaney, Princeton, Princeton University Press, 1992, p. 115-133 ; P. W. van der Horst, "Sortes : Sacred Books », p. 160.

45. Respectivement Christodore, Anthologie Palatine, 2, 1, 311-350 et 38-40.

46. Pour les citations d'auteurs païens dans les ouvrages chrétiens, voir par exemple Nicole Zeegers-Vorst, Les citations des poètes grecs chez les apologistes chrétiens du II siècle, Louvain, Bibliothèque de l'Université, 1972. Voir également l'étude de S. Morlet, Les chrétiens et la culture : conversion d'un concept, 
Homère et Platon constituant le couple divin par excellence en tant que précurseurs de la religion chrétienne. Selon plusieurs auteurs, qui entendent composer une symphonie « christianisante » des savoirs, les sagesses grecque et barbare s'inscrivent dans une théologie de l'histoire qui culmine dans la religion chrétienne et plonge ses racines dans la plus ancienne sagesse, la sagesse hébraïque : la vérité est une source de sagesse à laquelle Grecs et Barbares ont puisé ${ }^{47}$. Or, cette symphonie n'est pas toujours totale. En effet, les païens ont parfois mal interprété leurs sources, le rapprochement doctrinal et la sacralisation biographique de certaines figures impliquant souvent une manipulation textuelle. Ces figures ne peuvent, quoi qu'il en soit, être mises sur le même plan que les véritables figures prophétiques de la tradition biblique.

Dans cette perspective, on comprend mieux le phénomène de sacralisation chrétienne de la Sibylle et de Virgile, que l'on peut observer dans un discours attribué à l'empereur Constantin, connu comme l'Oratio Constantini ad sanctorum coetum. On y assiste à la métamorphose de la Sibylle, qui passe du statut de prophétesse païenne à celui de prophétesse du véritable dieu, celui des chrétiens ${ }^{48}$. Quant à Virgile, sa «conversion» apparaissait déjà dans l'exégèse chrétienne de la Quatrième Églogue. De plus, d'autres figures empruntées à des systèmes orientaux ont été resémantisées par les sources chrétiennes, comme c'est le cas de Zoroastre, un sage dont la culture hellénique s'est fortement inspirée. Cette figure, suspendue entre mythe et histoire, a connu un remarquable succès au cours des siècles qui ont suivi, et en particulier à la Renaissance ${ }^{49}$, où elle a été très largement reprise.

$I^{e r}$-VI $I^{e}$ siècle, Paris, Les Belles Lettres, 2016 et ici l'article de S. Morlet, notamment sur la symphônia des auteurs hellènes avec l'Écriture (par exemple chez Eusèbe).

47. Concernant la fiction de la «philosophie barbare» chez les Pères : les Hébreux ont reçu de Dieu-Logos la vérité, par la Loi et les Prophètes, mais de façon voilée. Les chrétiens, leurs héritiers, ont le privilège de pouvoir revendiquer le noble titre de «barbares » (plus ancien que la philosophie grecque) et grâce au Christ et à l'Évangile, ils ont la possibilité d'avoir accès à la révélation pleine et entière. Pour cette idée, voir Clément, Eusèbe de Césarée et Théodoret, par exemple, Aff. VIII 2, p. 310, 17 - 3 p. 311, 9 Canivet (VIII 2, p. 484, 4- p. 486, 14 Scholten).

48. Ce sera le sujet de l'article de Jean-Michel Roessli, à paraître ultérieurement.

49. Sur la réception de la figure de Zoroastre au fil des siècles, voir l'étude de Michael Stausberg, Faszination Zarathushtra : Zoroaster und die Europäische Religionsgeschichte der Frühen Neuzeit, t. 1-2, Berlin/New York, De Gruyter, 1998. 
Pour conclure, on se trouve face à une sorte de re-sémantisation du savoir antique et à une nouvelle mise en scène qui se réalise au niveau textuel et visuel. La «salle commune » (homakoeion) de Pythagore peut dès lors préfigurer l'Église chrétienne ${ }^{50}$ et les sapientes païens font ainsi figure de producteurs de textes oraculaires dans des recueils oraculaires chrétiens ${ }^{51}$.

Cette rencontre entre différents «systèmes » de pensée s'est, en outre, réalisée tantôt de manière divergente, tantôt de manière convergente. Deux ensembles de mosaïques tardives, qui datent du IV $V^{e}$ siècle, découvertes l'une à Apamée-sur-l'Oronte, l'autre à Nea Paphos (Chypre), témoignent de la manière polémique et allusive dont étaient employées les images qui faisaient référence aux mythes grecs et aux sages de la tradition hellénique. Ces mosaïques cachent un message néoplatonicien qui révèle un lien très fort entre images et textes. Néanmoins, outre cette divergence, ces allusions polémiques,

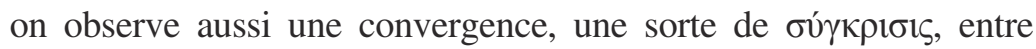
figures sacrées païennes, hébraïques et chrétiennes, comme dans

50. Clément d'Alexandrie, Stromates, I 15, 66, 2 Mondésert : «il (scil. Pythagore) fréquenta aussi les plus distingués des Chaldéens et des Mages, et ce que nous appelons aujourd'hui Église est préfiguré par sa "salle commune" » (tr. Caster). Sur les enseignements dispensés par des poètes grecs, et qu'eux-mêmes avaient reçus de prophètes juifs de manière détournée, cf. également Clément d'Alexandrie, Stromates, V 4, 24, 1-2 et pour les philosophes cf. Ibid., V 5, 29, 3-5 Le Boulluec.

51. Dans la Théosophie, les textes des sages abordent trois thèmes : 1. l'attaque contre le culte des statues ; 2 . le divin ; 3 . la nature humaine. Leur sagesse s'étend donc à des sujets rituels, éthiques et théologiques. Il reste des traces de textes poétiques et de maximes philosophiques de certains des sages païens cités, plus précisément d'Héraclite (67, 68, 69, 74 Erbse = II 15, 16, 17, 22 Beatrice), de Diogène le philosophe (70 E. = attribué à Diagore chez II $18 \mathrm{~B}$.), des passages de Platon ou attribués à Platon (une maxime $60 \mathrm{E}$. = II $8 \mathrm{~B}$., un passage tiré du Second Alcibiade 143a1-2 en $40 \mathrm{E}$. = II 7 B.), de la deuxième lettre à Denys (63 E. = II 11 B.), la célèbre maxime de Socrate sur la sagesse (62 E. = II 10 B.), un passage d'Antisthène (90 E. = II 30 B.), deux de Timon de Phlionte $(91 \mathrm{E} .=$ II 31 B.), un passage de Syrianus (50 E. = I 51 B.). Le philosophe Porphyre est cité comme source de deux oracles théologiques : l'un sur le dieu immortel, l'autre qui est une exhortation à adresser l'intellect à dieu (respectivement : 27-29 E.= I 24-26 B. ; 30 E. = I 29 B.). Dans un autre passage (65 E. = II 13 B.), il s'agit d'une maxime philosophique de Porphyre (cf. aussi l'anecdote de 85 E. =II 25 B.). Ces derniers oracles dérivent vraisemblablement de la Philosophia ex oraculis haurienda. Parmi les citations de poètes, on compte : Pindare (79 E. = II 23 B.), Simonide (84 E. = II 24 B.), Euripide (86 E. = II 26 B.) et Ménandre (87, 88, 89 E. $=$ II $27,28,29$ B.). On a aussi un passage qui concerne Denys, tyran de Syracuse $(71,72,73 \mathrm{E} .=$ II 19, 20, 21 B. $)$ et un préambule sur Jamblique comme élève de Porphyre (66 E. =I 14 B.). 
le célèbre cas du laraire (lararium) sur lequel Alexandre Sévère accomplissait des sacrifices en l'honneur des meilleurs empereurs divinisés et d'âmes saintes comme celles d'Apollonios de Tyane, du Christ, d'Abraham et d'Orphée ${ }^{52}$. Évoquons à ce propos une pratique des Carpocratiens - une secte gnostique - qui réalisaient des images, peintes ou créées à partir d'autres matériaux, du Christ et des philosophes du monde, à savoir Pythagore, Platon et Aristote ${ }^{53}$. Enfin, Moïse, Apollonios de Tyane et Hermès sont mentionnés dans la Théosophie de Tübingen - un recueil d'oracles composé par un auteur chrétien vers la fin du $\mathrm{V}^{\mathrm{e}}$ siècle $^{54}$ - où ils sont décrits comme les possesseurs d'un don qui les rapproche de la divinité. Dans des compilations oraculaires postérieures à la Théosophie, et indépendantes de cette dernière, publiées par Hartmut Erbse sous le titre de Thesauri minores ${ }^{55}$, les sages païens deviennent les prophètes

52. Hist. Aug. 29, 2 (datant de la fin du IV siècle); un autre laraire présentait le portrait de Virgile et une statue de Cicéron et d'Achille, ainsi que d'autres personnages. Voir Cécile Bertrand-Dagenbach, «Alexandre Sévère, ses héros et ses saints : ou quelques pieuses impiétés d'un bon empereur », Du héros païen au saint chrétien, dir. Gérard Freyburger, Laurent Pernot, Paris, Institut d'Études Augustiniennes, 1997, p. 95-103. Pour la reprise de la figure d'Orphée dans la pensée chrétienne voir Fabienne Jourdan, Orphée et les chrétiens : la réception du mythe d'Orphée dans la littérature chrétienne grecque des cinq premiers siècles. 1. Orphée, du repoussoir au préfigurateur du Christ: "réécriture d'un mythe à des fins protreptiques chez Clément d'Alexandrie », vol. 1, Paris, Les Belles Lettres, 2010, et Ead., Ibid. Pourquoi Orphée?, vol. 2, Paris, Les Belles Lettres, 2011.

53. Irénée, Contre les hérésies (Dénonciation et réfutation de la gnose au nom menteur), I, 25, 6 ; sur la secte, cf. aussi Hippolyte, Ref. VII, 32. On peut également évoquer le cas de l'épitaphe de Zénodote, chevalier de l'empereur, eques singularis, du temps de Marc Aurèle ou d'Hadrien, qui se présente comme une image de Socrate chez les Ausoniens. Son âme a rejoint le « séjour sacré des

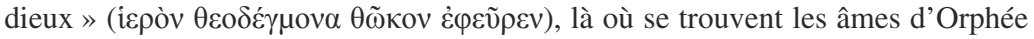
et de Platon (Anthologie Palatine VII 363, cf. CIL VI 32808 = Bücheler Carm. Ep. 474). Cf. aussi Ammien Marcellin XXI 14, 5 : Socrate, Pythagore, Apollonios de Tyane, Hermès Trismégiste et Plotin sont des hommes guidés par de puissants esprits (genii familiares).

54. Théosophie 44 E. (I 40 B.). Sur cet oracle, voir A. Busine, «Hermès Trismégiste, Moïse et Apollonius de Tyane dans un oracle d'Apollon », Apocrypha, t. 13, 2002, p. 227-243, et Lucia Maddalena Tissi, Gli oracoli degli dèi greci nella Teosofia di Tubinga. Commento e studio critico dei testi 12-54 Erbse, Alexandrie, Edizioni dell'Orso, 2018, p. 285-288.

55. Selon H. Erbse, ces recueils dérivent d'un archétype (Grundsammlung) qui aurait été rédigé avant Jean Malalas ( $\mathrm{vI}^{\mathrm{e}}$ siècle). Voir H. Erbse, Theosophorum Graecorum fragmenta, Stuttgart/Leipzig, Teubner, 1995², p. XXIV-XXv ; Anton von Premerstein, « Griechisch-heidnische Weise als Verkünder christlicher Lehre in Handschriften und Kirchenmalereien ", Festchrift in der Nationalbibliothek 
de la théologie chrétienne, en une véritable symphonie entre sagesse païenne et religion chrétienne.

\section{LES SAGES EN PERSPECTIVE}

Cette représentation des sages païens comme figures divinatoires persiste au cours des siècles pour atteindre son apogée à la Renaissance, alors que la culture grecque connaît un véritable renouveau. Nous nous pencherons ici sur deux cas de « métamorphose » des sages du passé en hommes divins, le premier étant un cas littéraire, le deuxième un dossier iconographique.

En 1540, Agostino Steuco publie un ouvrage en dix livres intitulé De perenni philosophia, visant à démontrer la concordance entre savoirs antiques et religion chrétienne, voire catholique, face aux réformes religieuses introduites en Europe $^{56}$. Steuco rassemble dans cette œuvre plusieurs oracles, des révélations multiples, autrement dit des paroles révélées, provenant de différentes sources, et qui sont mises en rapport avec la Révélation chrétienne ${ }^{57}$. On y trouve des textes orphiques, chaldaïques, sibyllins, hermétiques et théosophiques, ainsi que des citations tirées de Platon, Aristote, Proclus et intégrées à un système théologique chrétien. Cette stratégie de citation s'inscrit parfaitement dans une vision symphonique de savoirs hétéroclites, païens et chrétiens, mais elle témoigne aussi de la préparation

in Wien, Vienne, Österreische Staatdruckerei, 1926, p. 657-662, ici p. 664 ; contra Pier Franco Beatrice, Anonymi monophysitae Theosophia. An Attempt at Reconstruction, Leyde, Brill, p. LV-LVI, qui pense que les extraits oraculaires théosophiques édités par Erbse comme $\Sigma, \chi, \pi$ se trouvaient déjà dans la Théosophie.

56. Sur cette figure, voir Theodor Freudenberger, Augustinus Steuchus aus Gubbio. Augustinerchorherr und päpstlicher Bibliothekar, 1497-1548, und sein literarisches Lebenswerk, Munich, Aschendorffsche Buchhandlung, 1935.

57. Sur l'idée de prisca theologia et de philosophia perennis, voir Maria Muccillo, Platonismo ermetismo e prisca theologia: ricerche di storiografia filosofica rinascimentale, Florence, Leo S. Olschki, 1996, 1-72 (= Ead., «La "prisca theologia" nel "De perenni philosophia" di Agostino Steuco", Rinascimento, t. 28, 1988, p. 41-111); Charles B. Schmitt, «Prisca theologia e philosophia perennis : due temi del Rinascimento italiano e la loro fortuna », Il pensiero italiano del Rinascimento e il tempo nostro, dir. Giovannangiola Tarugi, Florence, Leo S. Olschki, 1970, p. 211-236, ici p. 211-213, 217-218 et Id., «Perennial Philosophy: From Agostino Steuco to Leibniz », Journal of the History of Ideas, t. 27, 1996, p. 505-532, ici p. 507-511. 
culturelle et de l'attitude intellectuelle de l'auteur. Les sages païens sont présentés ici, dans le sillage d'une tradition herméneutique, comme des précurseurs de la théologie chrétienne.

Quelques années plus tôt, dans une fresque du XvI ${ }^{\mathrm{e}}$ siècle $^{58}$ ornant la trapéza du monastère de la Grande Laure sur le mont Athos, douze sages (et non plus sept, en référence aux douze apôtres) sont représentés dans le motif de l'Arbre de Jessé. Il s'agit de : Socrate, Pythagore, $\dot{\eta}$ Ai $\alpha \lambda \eta \dot{\alpha} \alpha$ (?), Solon, Cléanthe, Philon (groupe A, à gauche de l'Arbre) et d'Homère, Aristote, Galien, Sibylle, Platon, Plutarque (groupe $\mathrm{B}$, à droite de l'Arbre). Chacun tient un rouleau portant des inscriptions, qui sont en fait des prophéties annonçant l'arrivée du Christ. Des œuvres similaires sont réalisées au cours du XVII ${ }^{\mathrm{e}}$ siècle dans des monastères en Grèce ${ }^{59}$, en Bulgarie ${ }^{60}$

58. La fresque a été réalisée dans les années 1527-1536. Un autre exemple que l'on peut évoquer est celui des Sibylles. Dans la cathédrale de Sienne, bâtie en 1480, les Sibylles livrant leurs oracles, tirés des Divinae Institutiones de Lactance, sont représentées comme des prophètes annonçant l'arrivée du Christ et d'une nouvelle époque de salut. Textes et images sont ainsi mis en relation dans un contexte prophétique. Un exemple antérieur est représenté par le portail royal de la cathédrale de Chartres et notamment par les voussures des portes latérales avec les représentations des sages de l'Antiquité, parmi lesquels on pense reconnaître Pythagore et Aristote : pour étudier l'iconographie de la cathédrale de Chartres, l'on pourra partir des bibliographies données dans le récent volume collectif $L a$ Grâce d'une cathédrale, Strasbourg, éditions de la Nuée Bleue, 2020 - recherche qui sort des limites de notre présentation.

59. Nikos A. Bees, «Darstellungen altheidnischer Denker und Autoren in der Kirchenmalerei der Griechen », Byzantinisch-Neugriechische Jahrbücher,

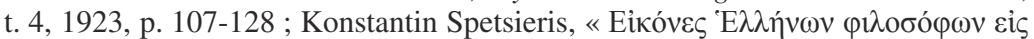

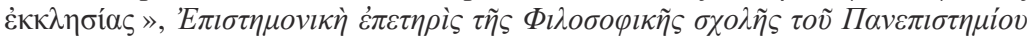
A $\theta \eta v \tilde{\omega} v$, t. 14, 1963-1964, p. 386-458.

60. On peut citer deux exemples de fresques bulgares : dans le réfectoire de Bačkovo, des fresques datant de 1623 représentent douze personnages, à savoir Aristophane, Odoneristos (ó $\Delta \tilde{\omega} v+\tau \rho \imath \sigma \mu \varepsilon ́ \gamma 1 \sigma \tau o \varsigma$ ?), Diogène, Ariklos (Arès ?), Kleomianos (Cléomède?), Sokrates / Sibylle, Platon, Plutarque, Hokiaros (Homère ?), Aristote, Galien. Dans le narthex de l'église Nativitas Christi d'Arbanasi, décoré par des fresques réalisées en 1681, les douze figures, insérées dans le motif de l'Arbre de Jessé, sont : Homère, Aristote, Galien, Sibylle, Platon, Plutarque, Lisitis (Lysias ?), Astakor (?), Solon, Zialigis (?), Pythagore, Socrate. Voir : André Grabar, La peinture religieuse en Bulgarie, Paris, Geuthner, 1928 ; Ivan Dujčev, «Die Begleitinschriften der Abbildungen heidnischer Denker und Schriftsteller in Bačkovo und Arbanasi », Jahrbuch der Österreichischen Byzantinischen Gesellschaft, t. 16, 1967, p. 205-209 (= Medioevo bizantinoslavo, vol. III, Altri saggi di storia politica e letteraria, Rome, Edizioni di storia e letteratura, 1971, p. 641-649) ; Id., « Heidnische Philosophen und Schriftsteller in der alten bulgarischen Wandmalerei », Opladen, Westdeuscher Vorlag, 1976, p. 7-22. En général, voir sur ce sujet la thèse de Sophia Zoumboulaki, L'Arbre 
et en Roumanie ${ }^{61}$. Les sages portent un nimbe à l'instar des saints chrétiens ${ }^{62}$. Les figures païennes, désormais privées de leur individualité, transformées en autorités figées, préfigurent la religion chrétienne. Enfin, dans un manuscrit d'Oxford, le Bodleianus Roe 5, aux fol. 149r-155v (xvII siècle), on trouve une version d'un opuscule faussement attribué à Athanase

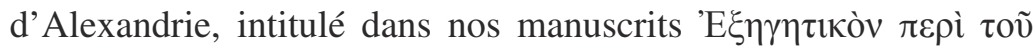

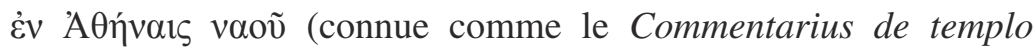
Athenarum $=C P G 2289)^{63}$. Les prophéties des Sept Sages annoncent l'avènement du Christ ${ }^{64}$. Les sages mentionnés sont : Titan, Bias, Solon, Chilon, Thucydide, Ménandre et Platon. Le manuscrit, qui date du début du $\mathrm{XVII}^{\mathrm{e}}$ siècle, est enluminé : les sages y sont représentés comme des prophètes tenant une banderole avec des inscriptions (qui sont en partie manquantes). Leurs portraits sont désormais identiques à ceux des prophètes de la tradition judéo-chrétienne.

de Jessé et la représentation des philosophes grecs et autres sages païens dans la peinture murale byzantine et post-byzantine, thèse de doctorat dirigée par Catherine Jolivet-Lévy (université Paris I) et soutenue le 9 juin 2015.

61. En Roumanie, dans le monastère de Voronet, toujours au sein du motif de l'Arbre de Jessé, on repère six figures : (le tsar) Aristote, Elin Platon, tsarina Siwila (la Sibylle), Elin Pilwogor (Pythagore), Elin Sokrat (Socrate), Elin Thglid (Thucydide). Les images datent de 1546. Voir : Ioan D. Stefanescu, L'évolution de la peinture religieuse en Bucovine et en Moldavie, depuis les origines jusqu'au XIX siècle. Nouvelles recherches, étude iconographique, Paris, Geuthner, 1928; Constantin I. Ciobanu, «Profetiile înteleptilor Antichitatii de la biserica Sfântul Gheorghe a mânastirii Voronet », Studii si cercetari de istoria artei-Seria arta plastica, t. 1, 2011, p. 11-32.

62. Ces prophéties dérivent des recueils $\mu, \tau, \delta$ de l'édition Erbse. C'est surtout

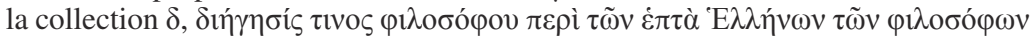

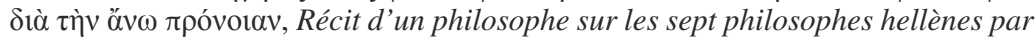
l'effet de la Providence d'en haut qui a exercé une influence. On y trouve : Diogène, Plutarque, Arès, Don le Trismégiste, Cléomède, Platon, Aristote, Homère.

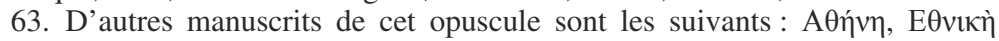

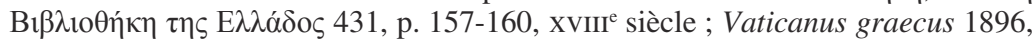
fol. $221^{\mathrm{r}}-227^{\mathrm{v}}$, $\mathrm{XVI}^{\mathrm{e}}$ siècle ; Vaticanus graecus 1198 , fol. $65^{\mathrm{r}}-66^{\mathrm{v}}$, $\mathrm{XVI}^{\mathrm{e}} \mathrm{s}$. (copie du

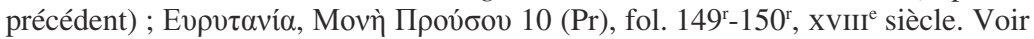
aussi Biblioteca Vallicelliana, Carte Allacci XCIV 40, XVII ${ }^{\mathrm{e}}$ siècle.

64. Sur la légende de Sept Sages, voir : Louis Bréhier, «La légende des sages païens à Byzance ", Mélanges d'histoire du Moyen-Âge dédiés à la mémoire de L. Halphen, Paris, 1951, p. 61-69; Armand Delatte, «Le déclin de la Légende des VII Sages et les Prophéties théosophiques », Musée Belge, t. 2, 1923, p. 97-111; A. von Premerstein, «Griechisch-heidnische Weise als Verkünder christlicher Lehre in Handschriften und Kirchenmalereien ». Voir aussi l'étude d'A. Busine, Les Sept Sages de la Grèce antique : transmission et utilisation d'un patrimoine légendaire d'Hérodote à Plutarque, Paris, De Boccard, 2002. 


\section{ENJEUX}

Dans l'horizon général qui vient d'être décrit, le présent recueil de textes est le fruit d'une journée d'étude que nous avons organisée le 12 juin 2018 à l'École Pratique des Hautes Études dans le cadre du LabEx Hastec (Laboratoire d'excellence « Histoire et anthropologie des savoirs, des techniques et des croyances »). Le but est de mettre en lumière le phénomène de sacralisation de sages païens entre les $\mathrm{II}^{\mathrm{e}}$ et $\mathrm{VI}^{\mathrm{e}}$ siècles de notre ère, à travers l'étude des portraits littéraires et figuratifs des sages en question, tout en cherchant à comprendre les raisons de l'emploi et de l'éventuelle « re-sémantisation » de ces figures par rapport au contexte culturel et historique originel.

Nous avons tenté de répondre à plusieurs questions dans le cadre de cette rencontre : est-ce que les discussions philosophiques, notamment celles qui sont inspirées par le médioplatonisme, jouent un rôle dans le processus de sacralisation des figures païennes? Quel usage les sources païennes et chrétiennes font-elles de cette sacralisation? Enfin, de quelle manière cette sacralisation a-t-elle influencé les arts figuratifs ? Les regards croisés et pluriels de différents chercheurs ont permis de mieux comprendre ce phénomène et le rôle de certaines stratégies dans l'histoire de la pensée et des religions.

Le dossier est construit selon une progression qui met en regard tradition philosophique «païenne » et littérature chrétienne, monde grec et monde latin, textes et images. Le parcours commence par deux études de cas internes à la tradition philosophique grecque : l'une porte sur la divinisation de Pythagore et la «pythagorisation » de Socrate et de Platon par le médioplatonicien Numénius au II $^{\mathrm{e}}$ siècle de notre ère ${ }^{65}$; l'autre est une enquête sur la sacralisation de Socrate dans le néoplatonisme tardif $\left(\mathrm{v}^{\mathrm{e}}-\mathrm{VI}^{\mathrm{e}} \text { siècle }\right)^{66}$. À l'univers néoplatonicien latin appartient l'œuvre de Martianus Capella ( $\mathrm{VI}^{\mathrm{e}}$ siècle), qui fait apparaître plusieurs figures de la philosophie et des sciences antiques aux noces de Philologie et de Mercure ${ }^{67}$. Après cette traversée de la littérature philosophique, l'on passe aux

65. Fabienne Jourdan, « Numénius et Pythagore. Pourquoi Numénius fait-il du pythagorisme le fondement du platonisme?».

66. Marco Donato, «Le divin Socrate. Autorité et "sacralisation" de Socrate dans le néoplatonisme tardif ».

67. Jean-Baptiste Guillaumin, « Savants et philosophes au mariage de Philologie : une sacralisation de figures du savoir antique chez Martianus Capella? ». 
textes chrétiens de l'Antiquité pour une analyse d'ensemble des modalités de la «sacralisation» des figures païennes dans cette littérature (en grec) ${ }^{68}$, avant d'étudier comment, dans un discours attribué à l'empereur Constantin - probablement écrit au IV siècle et transmis dans une version grecque - les figures de la Sibylle et de Virgile, christianisées, annoncent la vérité du Christ $^{69}$. Cet ensemble s'achève par l'évocation de Zoroastre, fondateur de la religion des Perses, qui a longtemps fasciné la culture européenne : réinterprétée dans l'univers gréco-latin, cette figure d'autorité est ici enrichie par l'examen de deux documents peu connus, chez Arnobe et dans le roman pseudo-clémentin ${ }^{70}$. Les images, mises en regard des textes, complètent l'enquête, et l'analyse de mosaïques romaines du $\mathrm{IV}^{\mathrm{e}}$ siècle, en Syrie et à Chypre, montre comment des figures païennes parfois préalablement christianisées ont été récupérées dans le contexte de la réaction antichrétienne, et chargées de significations néoplatoniciennes ${ }^{71}$. Le dossier s'achève, de façon décentrée, par une analyse qui montre comment le poids des «autorités » investies de sacralité, dans la philosophie néoplatonicienne de la fin de l'Antiquité, est néanmoins contrebalancé, chez nombre d'auteurs, par diverses stratégies de contournement qui dégagent une marge de manœuvre pour une certaine autonomie critique de la pensée philosophique ${ }^{72}$.

luciamaddalenatissi@gmail.com Philippe.Hoffmann@ephe.psl.eu

68. Sébastien Morlet, «La "sacralisation" des figures païennes dans les textes patristiques : le sens, les voies et les limites d'une notion ».

69. La contribution de Jean-Michel Roessli, «Christianisation de la Sibylle et de Virgile dans l'Oratio Constantini ad sanctorum coetum », dont l'ampleur aurait excédé les limites de ce fascicule, sera publiée prochainement ailleurs, mais s'insère dans le plan qui est ici exposé.

70. Chiara Ombretta Tommasi, «L'astre vivant. Quelques remarques sur le statut prophétique de Zoroastre dans la littérature gréco-romaine de l'Antiquité tardive ».

71. Janine Balty, «Mosaïques romaines du $\mathrm{IV}^{\mathrm{e}}$ siècle et réaction païenne : récupération et re-sémantisation des images ».

72. Adrien Lecerf, «Comment contourner une autorité : l'exemple du néoplatonisme post-plotinien ». 
Le présent numéro thématique est publié avec le concours du Laboratoire d'études sur les monothéismes et du Laboratoire d'excellence « Histoire et Anthropologie des Savoirs, des Techniques et des Croyances »

(ANR-10-LABX-85)

Le Laboratoire d'excellence HaStec, créé en 2011, est porté par l'École pratique des hautes études (Université PSL).

https://lem-umr8584.cnrs.fr

https://labexhastec-psl.ephe.fr/presentation-du-labex-hastec/ 